\title{
Longevidade de inflorescências de esporinha tratadas com sacarose e tiossulfato de prata ${ }^{1}$
}

\author{
TÂNIA FORSTER CARNEIRO²; FERNANDO L. FINGER ${ }^{3}$; \\ JOSÉ GERALDO BARBOSA ${ }^{3}$ e VANESSA R. DOS SANTOS ${ }^{2}$
}

\begin{abstract}
RESUMO
O presente trabalho teve como objetivo avaliar o efeito de soluções de condicionamento ("pulsing"), em inflorescências de esporinha (Consolida ajacis) visando ao aumento da vida em vaso pelo uso de sacarose, tiossulfato de prata (STS) e pela combinação destes elementos. As inflorescências foram colhidas, uniformizadas em tamanho e estádio de abertura das flores, e posteriormente colocadas em vasos por $30,60,90$ e 120 minutos com sacarose a 5\%, $10 \%, 15 \%$ e $20 \%$, ou em solução de STS a $0,2,0,4$, $0,6,0,8$ e $1,0 \mathrm{mM}$ e os respectivos controles com água destilada. Avaliaram-se os efeitos da combinação das soluções de $0,2,0,4,0,6,0,8$ e 1,0 mM de STS em combinação com sacarose a $5 \%$ por 30 minutos. A utilização de sacarose isoladamente não influenciou a vida em vaso ou a redução da abscisão das flores quando comparado com ao controle. O tratamento com STS, combinado ou não com sacarose a 5\%, aumentou a longevidade, a abscisão e murchamento das flores. Condicionamento das inflorescências com 1,0 mM de STS, combinado ou não com sacarose a $5 \%$ por 30 minutos, elevaram a longevidade de aproximadamente 8 dias (controle) para o mínimo de 15 dias e reduziu a abscisão das flores de $66,9 \%$ para valores inferiores a $17,5 \%$.
\end{abstract}

Palavras-chave: flor de corte, "“pulsing”, vida de vaso, abscisão.

\section{ABSTRACT
Longevity of Consolida ajacis inflorescences influenced by pulsing with sucrose and silver tiosulfate

The present work had the objective to evaluate the influence of silver tiosulfate (STS) in combination or not with sucrose on Consolida ajacis inflorescence vase life. The inflorescences were standardised in length and flower opening and than pulsed for 30, 60, 90 and 120 minutes in 5\%, 10\%, $15 \%$ and $20 \%$ sucrose or with $0.2,0.4,0.6,0.8$ and $1.0 \mathrm{mM}$ STS, and the control treatments with distilled water. In a second set of experiments the inflorescences were pulsed with $0.2,0.4,0.6,0.8$ and 1.0 mM STS combined with $5 \%$ sucrose for 30 minutes. Treatment with sucrose did not affect the vase life or flower abscission, when compared with the control. Pulsing the inflorescences with STS, in combination or not with $5 \%$ sucrose, were effective in extending the flower vase life and reducing flower abscission and wilting. Pulsing the inflorescences with $1.0 \mathrm{mM}$ of STS mixed or not with 5\% sucrose for 30 minutes increased flower longevity from approximately 8 days (control) to about 15 days, and fell down the abscission from $66.9 \%$ to values bellow to $17.5 \%$.

Key words: cut flower, pulsing, vase life, abscission.

\footnotetext{
${ }^{1}$ Extraído da Tese de Mestrado da primeira autora, bolsa concedida pela CAPES.

2 Departamento de Biologia Vegetal, UFV, 36571-000, Viçosa (MG).

${ }_{3}^{3}$ Departamento de Fitotecnia, UFV, 36571-000, Viçosa (MG) E-mail: ffinger@ufv.br
} 


\section{INTRODUÇÃO}

A espécie em estudo Consolida ajacis Nieuwl. pertence à família das ranunculáceas e é conhecida como esporinha, em razão do cálice floral em forma de espora. As inflorescências são vistosas, do tipo terminal e ereta, e as flores apresentam tonalidade roxa, lilás, rosa ou branca (LORENZI \& SOUZA, 1999). A utilização da esporinha como flor de corte é limitada, uma vez que, colhidas, sofrem rápido desenvolvimento do processo de abscisão e senescência das flores (FINGER et al., 2001). A senescência das flores de corte, segundo REID \& WU (1992), é determinada pela redução na absorção de água, murchamento e formação da camada de abscisão nas pétalas ou flores. A senescência de Lathyrus é caracterizada por sintomas de murchamento das pétalas e flores, geralmente a partir do segundo dia após a colheita (ISHIHARA et al., 1991), seguido da abscisão dos botões ou das pétalas (OHKAWA et al., 1987). Um dos principais fatores responsáveis pela abscisão das pétalas e flores é o aumento das taxas de produção de etileno (VAN DOORN \& STEAD, 1997), o qual estimula a formação de enzimas hidrolíticas e formação da zona de abscisão, como observado em flores de Limonium (DOI \& REID, 1995).

Para se obter maior longevidade e manter a qualidade das flores são necessárias práticas adequadas de pós-colheita, e o tratamento com soluções de condicionamento ou "pulsing" imediatamente após a colheita, contendo carboidratos ou inibidores da ação do etileno, podem aumentar a vida de vaso de diversas flores (NOWAK \& RUDNICK, 1990).

A sacarose é o carboidrato mais utilizado nas soluções de condicionamento, já que melhora a absorção de água pelas hastes e flores, e é utilizada como substrato para a manutenção da atividade respiratória (VAN DOORN, 2001). A sacarose utilizada em "pulsing" ou em solução de vaso é eficiente em aumentar a longevidade de diversas flores de corte, como observado em Lathyrus (ICHIMURA \& HIRAYA, 1999), Sandersonia (EASON et al., 1997), Gypsophyla (VAN DOORN \& REID, 1992), Eustoma (ICHIMURA et al., 1998), Anthyrium (ICHIMURA \& HISAMATSU, 1999) e Lilium (SANTANA et al., 1999). Em flores de Strelitzia reginae e Antirrhinum majus a aplicação de sacarose, além de estender a vida de vaso, promoveu a abertura dos floretes (FINGER et al., 1999; ICHIMURA \& HISAMATSU, 1999).
O íon de prata é utilizado na preservação de flores e plantas ornamentais principalmente na forma de solução de "pulsing", para reduzir os efeitos adversos da ação do etileno. A forma preferencial de utilização da prata é como tiossulfato de prata (STS)$\left[\mathrm{Ag}\left(\mathrm{S}_{2} \mathrm{O}_{3}\right)_{2}{ }^{-3}\right]$ por ser mais móvel e menos fitotóxico que o nitrato de prata $\left(\mathrm{AgNO}_{3}\right)$ (VAN ALTVORST \& BOVY, 1995). A aplicação de STS, na concentração de $0,5-1,0 \mathrm{mM}$ por 30 minutos a 1 hora, reduz o murchamento das pétalas e abscisão de flores que respondem à presença do etileno, como Lathyrus (ICHIMURA \& HIRAYA, 1999), Petunia (BOROCHOV et al., 1997), Gypsophila (NEWMAN et al., 1998) e Torenia (GOTO et al., 1999).

Embora muitas substâncias possam ser utilizadas na conservação de flores de corte, a composição das soluções e o tempo de aplicação pós-colheita variam entre as espécies. FINGER et al. (2001) observaram que a utilização de sacarose ou de STS em solução de "pulsing" prolongou a longevidade da esporinha, porém há necessidade de se realizarem novos experimentos para avaliar as interações entre tempo de aplicação, a concentração dos componentes e a presença de possível sinergismo entre a sacarose e STS sobre a senescência e abscisão das flores de esporinha. O presente trabalho teve como objetivos estabelecer soluções de "pulsing" específicas para a espécie C. ajacis, avaliar a eficiência da sacarose, tiossulfato de prata (STS) e a combinação entre eles sobre a vida da planta em vaso.

\section{MATERIAL E MÉTODOS}

As inflorescências de esporinha foram colhidas no campo de cultivo do Departamento de Fitotecnia da Universidade Federal de Viçosa, pelo período da manhã. O ponto de colheita das inflorescências estabelecido foi: hastes com $1 / 3$ das flores da inflorescência em fase botão, 1/3 em fase de abertura floral e $1 / 3$ das flores completamente abertas e cor das pétalas definida, conforme FINGER et al. (2001). As inflorescências foram transportadas para o laboratório, selecionadas, uniformizadas em tamanho, 40 a $50 \mathrm{~cm}$ de comprimento e dividas ao acaso para os diferentes experimentos.

Diariamente foram avaliados a taxa de abscisão das flores e o murchamento das pétalas por inflorescência. A vida de vaso das inflorescências foi considerada terminada quando o mínimo de $50 \%$ das flores haviam sofrido abscisão ou murchado (FINGER et al., 2001), conforme escala dos estádios 
de deterioração e senescência: Nota 0 - excelente turgidez das pétalas e rigidez da haste floral, ausência de descoloração das pétalas, ausência de abscisão de pétalas ou flores, aparência na colheita das inflorescências; Nota 1 - boa turgidez das pétalas e rigidez da haste floral; florescimento uniforme, máximo de $25 \%$ de murchamento com início de descoloração das pétalas, ausência de abscisão das pétalas ou flores; Nota $2-25 \%$ a $50 \%$ de murchamento das pétalas com descoloração aparente das pétalas, menos que $50 \%$ de abscisão das flores; Nota 3 - mais de $50 \%$ de murchamento e descoloração acentuada das pétalas; mais de $50 \%$ de abscisão das flores, fim da longevidade comercial.

As inflorescências tiveram a base de suas hastes imersas em 5, 10, 15 e 20\% de sacarose por 30, 60, 90 e 120 minutos, e o controle com água destilada. Em um segundo experimento, as bases das hastes das inflorescências foram imersas por 30, 60, 90 e 120 minutos com 0,$2 ; 0,4 ; 0,6 ; 0,8$ e $1,0 \mathrm{mM}$ de tiossulfato de prata e o respectivo controle. Num terceiro experimento, as hastes foram pulsadas por 30 minutos com 0,$2 ; 0,4 ; 0,6 ; 0,8$ e 1,0 mM de STS, combinados com sacarose a $5 \%$ e o controle com água destilada. Posteriormente, as inflorescências foram transferidas para vasos com água destilada e mantidas a temperatura de $24 \pm 1{ }^{\circ} \mathrm{C}$, umidade relativa de $60 \pm 20 \%$ e intensidade luminosa de $10 \mu \mathrm{mol} \mathrm{m} \mathrm{m}^{-2} \mathrm{~s}^{-1}$. A renovação da água destilada dos vasos foi realizada a cada dois dias.

Cada tratamento foi composto por 4 repetições com 5 hastes/vaso. Os dados foram interpretados por meio de análise de variância e regressão, utilizando-se o teste de "F", no nível de 5\% de probabilidade. As médias dos tratamentos foram comparadas com a do controle utilizando-se o teste de Dunnet, no nível de $5 \%$ de probabilidade.

\section{RESULTADOS E DISCUSSÃO}

Os principais sintomas da senescência das inflorescências de esporinha após a colheita das hastes foram a abscisão, seguida do murchamento das pétalas e flores. STEAD \& VAN DOORN (1994) verificaram que, em algumas espécies de flores, a abscisão das pétalas ocorre ainda quando estas estão túrgidas, geralmente em poucas horas após a abertura floral, como ocorre em Linum perene e Helianthemum vulgare, e, também, que o intervalo de tempo entre a dissolução da parede celular e a abscisão das flores varia entre as espécies. Essa variação no tempo pode ocorrer em razão da desidratação completa ou parcial das pétalas, como obser-

Tabela 1. Valores médios da abscisão e do murchamento das flores (\%) de flores de esporinha (Consolida ajacis Nieuwl.) após“"“pulsing” com 0,2; 0,4; 0,6;0,8 e 1,0 mM de STS, com 5, 10, 15 ou 20\% de sacarose por 30, 60, 90 e 120 minutos e controles, e "pulsing" com 0,2; 0,4; 0,6;0,8 e 1,0 mM de tiossulfato de prata combinado com $5 \%$ de sacarose por 30 minutos em função do número de dias após a colheita

Tratamentos

Dias após a colheita

\begin{tabular}{lllllll}
\hline 2 & 4 & 6 & 8 & 10 & 12 & 14
\end{tabular}

\begin{tabular}{|c|c|c|c|c|c|c|c|}
\hline \multirow[b]{2}{*}{ Sacarose } & \multicolumn{7}{|c|}{ Abscisão (\%) } \\
\hline & 0 & $0^{*}$ & $58,3^{*}$ & $71,7^{\text {ns }}$ & - & - & - \\
\hline $\mathrm{STS}^{* *}$ & 0 & $0^{*}$ & $0^{*}$ & $0^{*}$ & $8,7^{*}$ & $13,6^{*}$ & $19,0^{*}$ \\
\hline STS + sacarose & 0 & $0^{*}$ & $0^{*}$ & $0^{*}$ & $8,9^{*}$ & $15,2^{*}$ & $21,9^{*}$ \\
\hline \multirow[t]{2}{*}{ Controle } & 0 & 18,3 & 68,4 & 73,2 & - & - & - \\
\hline & \multicolumn{7}{|c|}{ Murchamento (\%) } \\
\hline Sacarose & 0 & 0 & $5,6^{*}$ & $82,3^{\text {ns }}$ & - & - & - \\
\hline STS & 0 & 0 & $0^{*}$ & $0^{*}$ & $7,8^{*}$ & $28,6^{*}$ & $83,7^{*}$ \\
\hline STS + sacarose & 0 & 0 & $0^{*}$ & $6,8^{*}$ & $8,6^{*}$ & $35,0^{*}$ & $85,2^{*}$ \\
\hline Controle & 0 & 0 & 53,3 & 94,0 & - & - & - \\
\hline
\end{tabular}

${ }^{n s}$ não significativo ou *significativo a $5 \%$ de probabilidade, pelo teste de Dunnet.

${ }^{* *}$ STS: tiossulfato de prata. 
vado em híbridos de rosas (VAN DOORN \& SCHRODER, 1995). As flores das famílias Geraniaceae, Labiatae, Ranunculaceae, Rosaceae e Scrophulariaceae, geralmente apresentam abscisão das pétalas durante a evolução da senescência (VAN DOORN \& SCHRODER, 1995). Como membro da família das Ranunculaceae, as esporinhas apresentam abscisão das flores como primeiro sintoma da senescência (WOLTERING \& VAN DOORN, 1988) semelhante aos sintomas observados nesse experimento.

Hastes de esporinha tratadas com sacarose aplicada na forma de "pulsing" reduziu as taxas de abscisão e o murchamento das flores até o sexto dia após a colheita, cujas hastes tratadas com sacarose apresentaram 58,3\% de abscisão e 5,6\% de murchamento, significativamente inferiores ao controle, porém ao oitavo dia após a colheita, a abscisão e o murchamento das flores foram semelhantes àquelas observadas para o tratamento controle (Tabela 1). ICHIMURA \& HISAMATSU (1999) observaram em Antirrhinum majus que a manutenção das hastes em solução de vaso contendo $5 \%$ de sacarose foi eficiente em reduzir o início da produção climatérica de etileno, retardando, em conseqüência, a senescência das flores. A redução inicial da abscisão e do murchamento das flores de esporinha, promo-

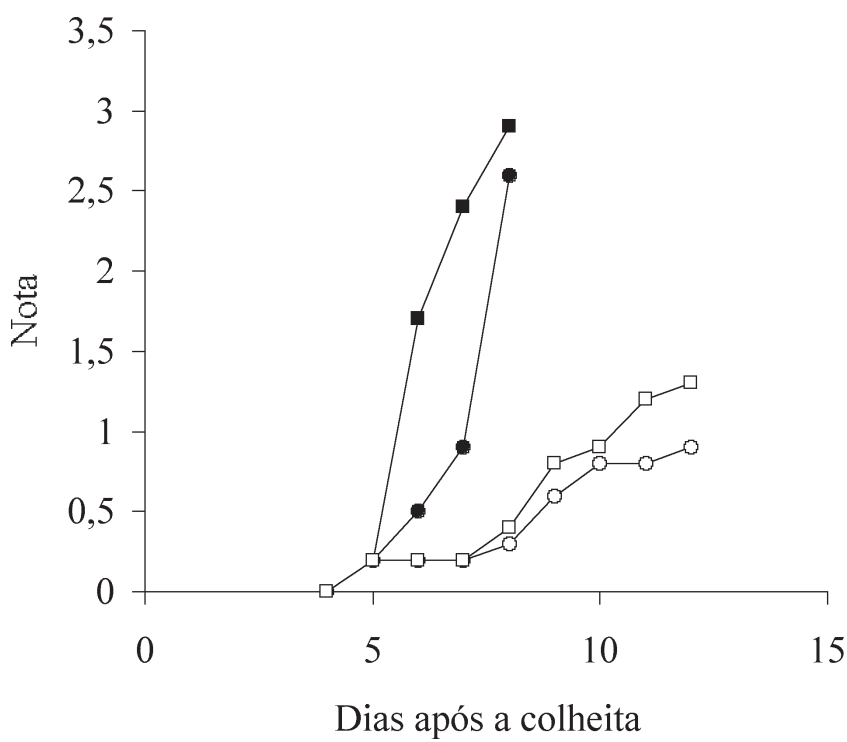

Figura 1. Evolução da deterioração das inflorescências de esporinha (Consolida ajacis Nieuwl.), tratadas em "pulsing" por 30 minutos com $5 \%$ de sacarose (๑),0,2;0,4;0,6;0,8 e 1,0 mM de tiossulfato de prata isoladamente $(\mathrm{O})$, ou combinado com 5\% ( $\square$ ) de sacarose, e controle vida pela sacarose, não se refletiu em aumento da longevidade de aproximadamente 8 dias observada para as inflorescências controle (Figura 1). Novos experimentos devem, portanto, ser realizados tendo em vista verificar se a aplicação de sacarose em solução de vaso não seria mais eficiente para prolongar os efeitos benéficos temporários observados pelo uso da sacarose na forma de "pulsing".

O condicionamento das hastes com STS, independentemente do tempo de aplicação, e quando utilizado em conjunto ou não com $5 \%$ de sacarose por $30 \mathrm{mi}-$ nutos, prolongou a longevidade das flores por reduzir significativamente a abscisão e o murchamento das pétalas até 14 dias após a colheita (Tabela 1). Em Lathyrus odoratus foi observado que o "pulsing" com sacarose associada ao STS estendeu a longevidade quando comparado com o tratamento das flores com sacarose ou STS isoladamente (ICHIMURA \& HIRAYA, 1999). Em Gladiolus, porém, não foi observado nenhum efeito sinérgico na combinação da sacarose com STS no aumento da abertura das flores e conseqüentemente na longevidade final das inflorescências (SEREK et al., 1994).

O tratamento de "pulsing" com 0,$2 ; 0,4 ; 0,6$; 0,8 e $1,0 \mathrm{mM}$ de STS, combinado ou não com $5 \%$ de sacarose, estendeu significativamente a longevidade e reduziu a abscisão das flores (Tabela 2).

Tabela 2. Longevidade e abscisão das flores de esporinha (Consolida ajacis Nieuwl.), tratadas em "pulsing" por 30 minutos com 0,$2 ; 0,4 ; 0,6 ; 0,8$ e $1,0 \mathrm{mM}$ de tiossulfato de prata isoladamente, ou combinado com $5 \%$ de sacarose, e controle com água destilada

\begin{tabular}{lcc}
\hline Tratamentos & Longevidade & Abscisão \\
\hline & dias & $\%$ \\
Controle & 7,9 & 66,9 \\
0,2 mM STS & $14,7^{*}$ & $25,0^{*}$ \\
$0,4 \mathrm{mM} \mathrm{STS}$ & $14,9^{*}$ & $22,2^{*}$ \\
$0,6 \mathrm{mM} \mathrm{STS}$ & $15,0^{*}$ & $19,2^{*}$ \\
0,8 mM STS & $15,5^{*}$ & $17,9^{*}$ \\
1,0 mM STS & $15,7^{*}$ & $17,5^{*}$ \\
0,2 mM STS + 5\% sacarose & $11,5^{*}$ & $30,7^{*}$ \\
0,4 mM STS + 5\% sacarose & $12,7^{*}$ & $31,3^{*}$ \\
0,6 mM STS + 5\% sacarose & $13,4^{*}$ & $21,4^{*}$ \\
$0,8 \mathrm{mM} \mathrm{STS}+5 \%$ sacarose & $14,7^{*}$ & $17,3^{*}$ \\
1,0 mM STS + 5\% sacarose & $15,0^{*}$ & $16,8^{*}$ \\
\hline
\end{tabular}

"Significativo a 5\% de probabilidade, pelo teste de Dunnet. ${ }^{* *}$ STS: tiossulfato de prata. 
A elevação da concentração de STS entre 0,2 e $1,0 \mathrm{mM}$ aumentou progressivamente a longevidade com concomitante redução na taxa de abscisão das flores (Tabela 2), resultando, portanto, em melhor aparência final das inflorescências para a concentração de 1,0 mM de STS, como evidenciada aos 15 dias após a colheita (Figura 2). Em outras flores, o tratamento com STS mostrou-se efetivo em reduzir a abscisão das flores de Calceolaria, de brácteas de Bougainvillea e das pétalas de Pelargonium e Torenia (CAMERON \& REID, 1983; GOTO et al., 1999). A presença de elevadas taxas de produção de etileno está associada com a rápida senescência e abscisão dos órgãos florais. Nestas flores, a presença de inibidores da produção ou ação de etileno é geralmente eficiente em reduzir a deterioração associada à presença do etileno (SEREK \& REID, 1992; SEXTON, 1994). Em esporinha, o tratamento em "pulsing" com STS isoladamente, nas formulações utilizadas, foram eficazes em aumentar a vida em vaso e inibir o processo de senescência e abscisão das flores, provavelmente pelo bloqueio da ação do etileno exercida pela presença do íon $\mathrm{Ag}^{+}$. A adição de $5 \%$ de sacarose à solução de STS não modificou a tendência dos resultados da longevidade e abscisão das flores quando comparado com as hastes tratadas com STS isoladamente (Tabela 2). Contrariamente, em flores de Lathyrus odoratus, a combinação de STS com sacarose em "pulsing" foi mais efetiva em estender a vida de vaso que o STS isoladamente, em

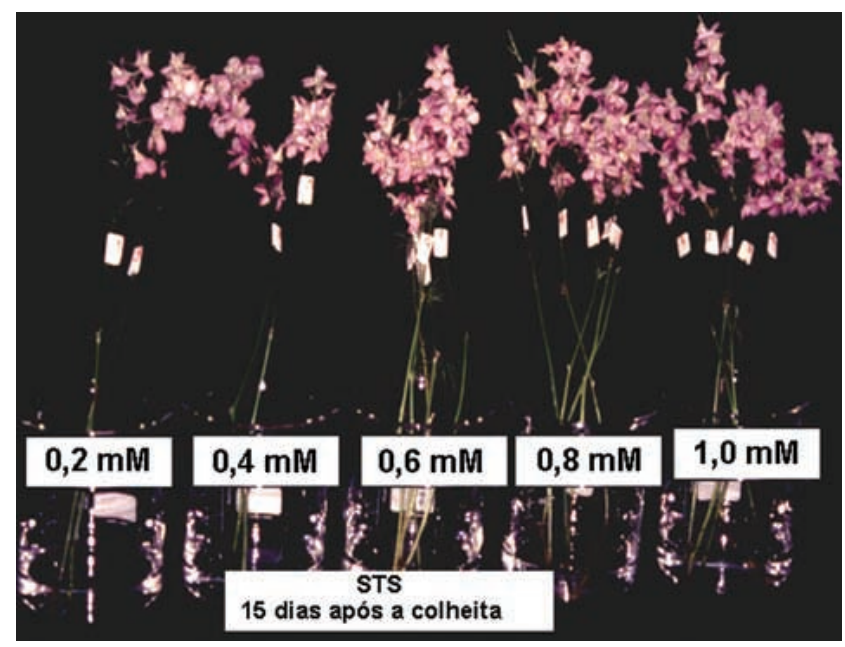

Figura 2. Aparência das inflorescências de esporinha (Consolida ajacis Nieuwl.) tratadas com 0,2; 0,4; 0,6; 0,8 e $1,0 \mathrm{mM}$ de tiossulfato de prata em "pulsing" por 30 minutos aos 15 dias após a colheita. razão da ação positiva da sacarose em inibir a produção de etileno pelas flores (ICHIMURA \& HIRAYA, 1999). A sacarose também se mostrou efetiva em aumentar a longevidade de Eustonia grandiflorum quando aplicada continuadamente na solução de vaso (ICHIMURA \& KORENAGA, 1998). Futuros experimentos, portanto, devem ser realizados para avaliar se a utilização de sacarose continuamente na solução de vaso poderia exercer maior controle sobre o curso da senescência em flores de esporinha.

\section{CONCLUSÕES}

1. A utilização de solução de "pulsing" com 5, 10,15 e $20 \%$ de sacarose por $30,60,90$ e 120 minutos não influenciou a longevidade final das inflorescências em vaso.

2. O tratamento de "pulsing" por 30 minutos com STS nas concentrações de 0,$2 ; 0,4 ; 0,6 ; 0,8$ e $1,0 \mathrm{mM}$, combinadas ou não com sacarose a $5 \%$, elevaram entre 1,49 e 1,99 vezes a vida de vaso das inflorescências e reduziram em 2,14 a 3,98 vezes a abscisão das flores.

\section{AGRADECIMENTOS}

À CAPES e ao CNPq, pela concessão das bolsas a Tânia F. Carneiro e Fernando L. Finger respectivamente.

\section{REFERÊNCIAS BIBLIOGRÁFICAS}

BOROCHOV, A.; SPIEGELSTEIN, H. \& PHILOSOPH, H.S. Ethylene and flower petal senescence: interrelationship with membrane lipid catabolism. Physiologia Plantarum, v.100, p.606-612, 1997.

CAMERON , A.C. \& REID, M.S. Use of silver tiosulfate to prevent flower abscission from potted plants. Scientia Horticulture, v.19, p.373-378, 1983.

DOI, M. \& REID, M.S. Sucrose improves the postharvest life of cut flower of a hybrid limonium. HortScience, v.30, p.1058-1060, 1995.

EASON, J.R.; VRÉ, S.D. \& HEYES, J.A. Physiological changes with Sandersonia aurantiaca flower senescence in response to sugar. Postharvest Biology and Technology, v.12, p.43-50, 1997.

FINGER, F.L.; CAMPANHA, M.M.; BARBOSA, J.G. \& FONTES, P.C.R. Influence of ethephon, silver thiosulfate and sucrose pulsing on bird-of-paradise vase life. Revista Brasileira de Fisiologia Vegetal, v.11, p.119-122, 1999. 
FINGER, F.L.; SANTOS, V.R.; MORAES, P.J. \& BARBOSA, J.G. Pulsing with sucrose and silver thiosulfate extend the vase life of Consolida ajacis L. Acta Horticulturae, v.543, p.63-67, 2001.

GOTO, R.; AIDA, R.; SHIBATA, M. \& ICHIMURA, K. Role of ethylene on flower senescence of Torenia. Journal of the Japanese Society for Horticultural Science, v.68, p.263-266, 1999.

ICHIMURA, K. \& HIRAYA, T. Effects of silver tiosulfate complex (STS) in combination with sucrose on the vase life of cut sweet pea flowers. Journal of the Japanese Society for Horticultural Science, v.68, p.23-27, 1999.

ICHIMURA, K \& HISAMATSU, T. Effects of continuous treatment with sucrose on the vase life, soluble carbohydrate concentrations, and ethylene production of cut Snapdragon flowers. Journal of the Japanese Society for Horticultural Science, v.68, p.61-66, 1999.

ICHIMURA, K. \& KORENAGA, M. Improvement of vase life and petal color expression in several cultivars of cut Eustoma flowers using sucrose with 8-hyrixyquinoline sulfate. Bulletin of the National Research Institute of Vegetables, Ornamental Plants and Tea, v.13, p.3139, 1998.

ICHIMURA, K.; SHIMAMURA, M. \& HISAMATSU, T. Role of ethylene in senescence of cut Eustoma flowers. Postharvest Biology and Technology, v.14, p.193-198, 1998.

ISHIHARA, Y.; OHKAWA., K. \& HYODO, H. Senescence of cut sweet pea flowers and ethylene production. Journal of the American Society for Horticultural Science, v.60, p.141-147, 1991.

LORENZI, H. \& SOUZA, H.M. Plantas ornamentais no Brasil, arbustivas, herbáceas e trepadeiras. 2.ed. Nova Odessa: Instituto Plantarum de Estudos da Flora Ltda., 1999. $967 \mathrm{p}$.

NEWMAN, J.P.; DODGE, L.L. \& REID, M.S. Evaluation of ethylene inhibitors for postharvest treatment of Gypsophila paniculata L. HortTechnology, v.8, p.5863, 1998.

NOWAK, J. \& RUDNICK, R.M. Postharvest handling and storage of cut flower, florists, greens and potted plants. Portland: Timber Press, 1990. 210p.

OHKAWA, K.; SUMITANI, K.; SATOL, S.; HAYASHI, I. \& YAMAMOTO, K. Effect of silver tiosulfate (STS) on the vase life and bud drop of winter flowering sweet pea (Lathyrus odoratus L.). Acta Horticulturae, v.210, p.223-230, 1987.

REID, M.S. \& WU, M.J. Ethylene and flower senescence. Plant Growth Regulation, v.11, p.37-43, 1992.

SANTANA, A.R.; BARBOSA, J.G.; FINGER, F.L. \& ROMEIRO, R.S. Longevidade de inflorescência de lírio condicionadas em sacarose. In: CONGRESSO BRASILEIRO DE FLORICULTURA E PLANTAS ORNAMENTAIS, 12., Jabotical, 1999 Anais... Jaboticabal: SBFPO, 1999. p.24.

SEREK, M. \& REID, M.S. Anti-ethylene treatments for potted Christmas cactus-efficacy of inhibitors of ethylene action and biosynthesis. HortScience, v.28, p.1180-1181, 1992.

SEREK, M.; JONES, R.B. \& REID, M.S. Role of ethylene in opening and senescence of Gladiolus sp. flowers. Journal of the American Society for Horticultural Science, v.119, p.1014-1019, 1994.

SEXTON, R. Abscission. In: PESSARAKLI M. (Ed.). Handbook of plant and crop physiology. New York: Marcel Decker, 1994. 640p.

STEAD, A.D. \& VAN DOORN, W.G. Strategies of flower senescence - a review. In: SCOTT, R.J. \& STEAD, A.D. (Eds.). Molecular and cellular aspects of plant reproduction. Cambridge: Cambridge University Press, 1994. p.215-237.

VAN ALTVORST, A.C. \& BOVY, A.G. The role ethylene in the senescence of carnation flowers, a review. Plant Growth Regulation, v.16, p.43-53, 1995.

VAN DOORN, W.G. Role of carbohydrates in flower senescence: a survey. Acta Horticulturae, v.543, p.179$183,2001$.

VAN DOORN, W.G. \& REID, M.S. Role of ethylene in flower senescence of Gypsophila paniculata L. Postharvest Biology and Technology, v.1, p.265-272, 1992.

VAN DOORN, W.G. \& SCHRODER, C. The abscission of Rose petals. Annals of Botany, v.76, p.539-544, 1995.

VAN DOORN, W.G. \& STEAD, A.D. Abscission of flowers and floral parts. Journal of Experimental Botany, v.48, p.821-837, 1997.

WOLTERING, E.J. \& VAN DOORN, W.G. van. Role of ethylene in senescence of petals-morphological and taxonomical relationships. Journal of Experimental Botany, v.39, p.1605-1616, 1988. 